\title{
Circulação extracorpórea com desvio veno-arterial e baixa pressão parcial de oxigênio
}

\author{
Mário Coli Junqueira de MORAES *,**, Domingos Junqueira de MORAES *,**, \\ Eduardo Sérgio BASTOS ${ }^{* * *}$, Henrique MURAD ***
}

Moraes M C J, Moraes D J, Bastos E S, Murad H - Circulação extracorpórea com desvio veno-arterial e baixa pressão parcial de oxigênio. Rev Bras Cir Cardiovasc 2001; 16(3): 251-61.

RESUMO: Objetivo: Esse trabalho divide-se em 2 partes, um estudo experimental para fundamentar a técnica de circulação extracorpórea com baixa pressão parcial de oxigênio e um estudo clínico, para mostrar a viabilidade de sua utilização em seres humanos.

Material e Métodos: No estudo experimental empregou-se a circulação extracorpórea em 20 cães divididos em 2 grupos de 10 animais. No grupo I, canulou-se separadamente a veia cava superior e, a seguir, a veia cava inferior, mantendo-se normais os batimentos cardíacos e a respiração, controlada com respirador e oxigênio puro. O sangue de cada veia cava depois de passar por um permutador de calor foi injetado na artéria femoral. A cada 30 minutos foram retiradas amostras de sangue da aorta acima do diafragma para determinação da gasometria. No grupo II, foi drenado o átrio direito e metade do sangue foi injetada na artéria pulmonar com outra bomba e recolhida pelo ventrículo esquerdo ao reservatório que é ao mesmo tempo, permutador de calor. O sangue, misturado na proporção de $50 \%$ venoso e $50 \%$ arterial foi reinjetado pela outra bomba na circulação arterial. O coração foi mantido fibrilando e a respiração controlada pelo respirador. No estudo clínico, os pacientes foram divididos em 2 grupos (Grupo A e B). O grupo A de 20 pacientes foi perfundido com sistema convencional, isto é, usando-se ar comprimido e oxigênio no oxigenador de membrana e alto $\mathrm{pO}_{2}$ arterial. No grupo $\mathrm{B}$, também com 20 pacientes, foi utilizado o oxigenador de membrana, oxigênio puro e desvio venoarterial, mantendo-se o desvio em torno de $40 \%$ a $50 \%$.

Resultados: No estudo experimental, em ambos grupos, do ponto de vista fisiológico, houve desvio de $50 \%$ do sangue venoso para a circulação arterial e fluxo de perfusão mantido alto $(100 \mathrm{ml} / \mathrm{kg} / \mathrm{min})$. Observou-se que o $\mathrm{pO}_{2}$ arterial em ambos grupos manteve-se entre $50 \mathrm{mmHg}$ e $100 \mathrm{mmHg}$ e a saturação venosa entre $50 \%$ e $70 \%$. Todos os animais acordaram no final da perfusão. No estudo clínico verificouse baixo $\mathrm{pO}_{2}$ arterial e fluxo de perfusão normal. Comparando-se os resultados clínicos constatou-se que não houve diferença de mortalidade nos 2 grupos, porém no grupo com baixo $\mathrm{pO}_{2}$ e desvio venoarterial o sangramento pós-operatório foi significativamente menor, utilizando-se três vezes menos hemoderivados. Além disso, não foi necessário o uso de misturador de gases.

DESCRITORES: Circulação extracorpórea, métodos. Pressão arterial, fisiologia. Oxigênio, fisiologia. Oxigenadores de membrana. Veias cava, cirurgia. Artéria pulmonar, cirurgia.

Trabalho realizado no Hospital Universitário Antônio Pedro da Universidade Federal Fluminense (UFF) e no Hospital Adventista Silvestre. Rio de Janeiro, RJ, Brasil.

Apresentado ao $27^{\circ}$ Congresso Nacional de Cirurgia Cardíaca. Rio de Janeiro, 23 a 25 de março de 2000.

* Do Hospital Universitário Antônio Pedro - UFF.

** Do Hospital Adventista Silvestre.

*** Do Hospital Universitário da Universidade Federal do Rio de Janeiro - UFRJ.

Endereço para correspondência: Mário Coli Junqueira de Moraes. Rua Ramon Franco, 66. Rio de Janeiro, RJ, Brasil. CEP 22290-290. Tel.: (21) 2295-5044. E-mail: drmoraes@ uninet.com.br 
Moraes M C J, Moraes D J, Bastos E S, Murad H - Circulação extracorpórea com desvio veno-arterial e baixa pressão parcial de oxigênio. Rev Bras Cir Cardiovasc 2001; 16(3): 251-61.

\section{INTRODUÇÃO}

É certo que a circulação extracorpórea determina alterações no sangue e nos tecidos perfundidos, gerando segundo a opinião de KIRKLIN et al. (1) um quadro geral de inflamação ou auto-agressão.

Inicialmente, reconhecida com denominações tais como, síndrome pós-perfusão, pulmão de bomba, passou posteriormente a ser conceituada como reação inflamatória geral do organismo. Esse conceito foi inicialmente proposto por KIRKLIN et al. (1). As manifestações clínicas se caracterizam por alterações funcionais muito ou pouco severas nas funções pulmonares, renais, acúmulo de líquido no espaço intersticial, febre, leucocitose, vasoconstricção e maior susceptibilidade a infecções.

Experimentalmente, nota-se que essas alterações são bem menos acentuadas na circulação assistida, onde o sangue também passa artificialmente pelos tubos de plástico, sendo impulsionado por uma bomba mecânica, entretanto, oxigenado pelo próprio pulmão do animal da experiência.

Apesar da maioria dos pacientes submetidos à operações cardíacas evoluir bem, boa parte da morbidade e certa percentagem na mortalidade podem ser atribuídas a circunstâncias não-fisiológicas da circulação extracorpórea com exclusão cardiopulmonar. De fato houve, em todos esses anos de pesquisa e aplicação clínica, melhora substancial dos oxigenadores e do sistema de monitorização, mas pouco se mudou no circuito.

A proposta do nosso trabalho visa à modificação deste circuito de perfusão com a introdução do conceito de desvio e mistura veno-arterial, usando o princípio da circulação fetal que resulta em transferência do oxigênio materno para a circulação sistêmica do feto.

Assim, o nosso trabalho tem por objetivo estudar um modelo experimental em que o débito circulatório durante a circulação extracorpórea, seja alto, próximo ao normal, porém com redução de $50 \%$ do fluxo pulmonar, determinando dessa forma uma queda no $\mathrm{pO}_{2}$ arterial, mas mantendo normal o débito sistêmico e em condições de normotermia.

O outro objetivo é estudar essa mesma situação em uma série clínica, comparando 2 grupos de 20 pacientes. Em um deles usando perfusão com $\mathrm{pO}_{2}$ alto e misturador de gases (oxigênio e ar comprimido) no oxigenador de membrana; no outro grupo, fazendo um desvio e mistura veno-arterial de $60 \%$ do sangue venoso pela membrana do oxigenador, com a manutenção do $\mathrm{pO}_{2}$ baixo e fluxo de perfusão normal, usando, dessa forma, somente oxigênio, sem necessitar de ar comprimido e portanto, de misturador de gases ("blender").

\section{MATERIAL E MÉTODOS}

\section{Estudo Experimental}

Foram estudados 20 cães, de raça mista ( 7 fêmeas e 13 machos), com peso variável entre $9 \mathrm{~kg}$ e $21,5 \mathrm{~kg}$ numa média de $13 \mathrm{~kg}$, oriundos do Instituto Municipal de Medicina Veterinária do Rio de Janeiro, já devidamente vacinados e submetidos a quarentena. Permaneceram eles no biotério da cirurgia experimental do Hospital Universitário Antônio Pedro - UFF e no biotério da cirurgia experimental do Hospital Adventista Silvestre, sendo operados no período de 10 de janeiro de 96 a 11 de junho de 97 e classificados para o presente estudo em 2 grupos de 10 cães:

- Grupo I: Circulação extracorpórea com coração batendo;

- Grupo II: Circulação extracorpórea com coração fibrilando.

\section{Grupo I}

Integraram este grupo 10 cães: 3 fêmeas e 7 machos, pesando entre $10 \mathrm{~kg}$ e $20 \mathrm{~kg}$ (média 12,5 $\mathrm{kg}$ ). Todos os animais foram submetidos à circulação extracorpórea com temperatura entre $34^{\circ} \mathrm{C}$ e $36^{\circ} \mathrm{C}$ e duração de 60 minutos.

Os cães foram anestesiados com tiopental sódico (4 mg/kg) e pancurôneo $(0,3 \mathrm{mg} / \mathrm{kg})$ na veia cubital do membro dianteiro esquerdo, aplicando-se nessa mesma veia soro Ringer Lactato lento. Foi realizada punção de artéria femoral esquerda sob visão direta para medir a pressão arterial média (PAM) e retirada de amostra de sangue para gasometria.

A ventilação foi mantida com intubação orotraqueal e respirador de pressão Takaoka ${ }^{\circledR}$, modelo Assistor 690. Toracotomia ântero-lateral direita pelo quarto espaço intercostal.

Os membros inferiores foram mantidos em extensão para que uma artéria femoral servisse de via de retorno arterial e a outra artéria femoral fosse utilizada para monitorização da pressão arterial média e coleta de amostras para gasometrias.

Mediu-se a temperatura retal com termômetro "Yelow Spring".

As canulações arterial e venosa foram realizadas com material próprio descartável.

Manteve-se, durante a toracotomia, rigorosa hemostasia com uso de bisturi elétrico. Preencheuse o circuito de circulação extracorpórea com expansor plasmático $(500 \mathrm{ml})$ e manitol $(50 \mathrm{ml})$. 
Moraes M C J, Moraes D J, Bastos E S, Murad H - Circulação extracorpórea com desvio veno-arterial e baixa pressão parcial de oxigênio. Rev Bras Cir Cardiovasc 2001; 16(3): 251-61.

Após abertura do pericárdio, a veia cava inferior foi canulada com tubo de material plástico ( $\mathrm{n}$ - 26 e n 28) através da aurícula direita e laçada com fita cardíaca. A artéria femoral direita ou esquerda foi canulada com cânula própria, para se fazer o retorno do sangue drenado pela veia cava no reservatório de cardioplegia. Iniciada a circulação extracorpórea, foi garroteada a veia cava inferior e o sangue todo desviado para o reservatório de onde, ao mesmo tempo, era bombeado para a artéria femoral do cão, permanecendo o mesmo nível no reservatório de cardioplegia sangüínea DMG.

Mantinham-se monitorizada a pressão arterial e a ventilação pulmonar. O animal continuou nessa situação durante 30 minutos.

Após esse tempo, a cânula posicionada na cava inferior era posicionada na cava superior e garroteada. Assim, todo o sangue da cava superior se desviava para o reservatório durante mais 30 minutos. Repetiam-se as mesmas dosagens de gases, bem como, a monitorização da pressão arterial média e o fluxo sangüíneo medido pela bomba de rolete.

\section{Grupo II}

O grupo II constituiu-se, como o anterior, de 10 cães: 6 machos e 4 fêmeas, pesando entre $9 \mathrm{~kg}$ e $21,5 \mathrm{~kg}$, com peso médio de $14 \mathrm{~kg}$. Em essência se manteve, do ponto de vista fisiológico, a mesma situação, ou seja, apenas a metade do sangue drenado das veias cavas passava pelo pulmão. A diferença principal é que, neste grupo, se injeta o sangue venoso na artéria pulmonar por meio de outra bomba de rolete, e calcula-se a metade do fluxo pela média obtida de cada veia cava nas experiências anteriores, isto é, $50 \mathrm{ml} / \mathrm{kg} / \mathrm{min}$ (Figura 1).

De modo geral, essa quantidade é que representa a metade do débito sistêmico.

Alguns minutos após iniciar a circulação extracorpórea, o coração é fibrilado artificialmente.

O sangue venoso injetado na artéria pulmonar por meio de outra bomba de rolete é recolhido, já oxigenado, pela ponta do ventrículo esquerdo e, por gravidade, vai-se juntar no mesmo reservatório. Daí, o sangue já misturado com o sangue do átrio direito é injetado na artéria femoral, mantendo a circulação sistêmica com fluxo total.

Como a mistura do sangue venoso e arterial se faz no reservatório, a dosagem de gases do sangue arterial pode ser realizada em qualquer ponto da linha arterial, ou na aorta torácica. A dosagem de gases do sangue venoso é retirada diretamente do átrio direito, e a pressão arterial é monitorizada

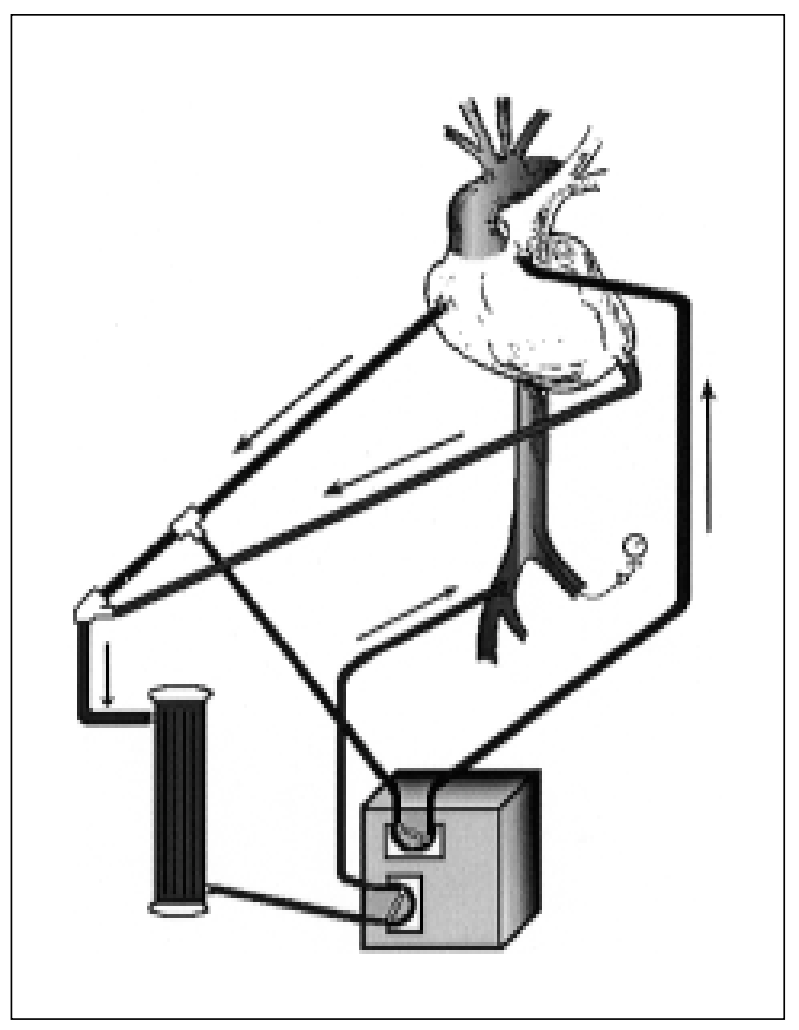

Fig. 1 - Esquema do grupo II, mostrando drenagem de átrio direito e ventrículo esquerdo com retorno às artérias femoral e pulmonar com duas bombas de rolete separadas.

constantemente. No final de 30 minutos, o coração é desfibrilado com cardioversão elétrica e, após 5 minutos, novamente posto a fibrilar. A perfusão é mantida por 60 minutos como no grupo $\mathrm{I}$.

\section{Estudo Clínico}

Foram estudados, retrospectivamente, 40 pacientes escolhidos ao acaso dentro de limites que variaram de 60 até 120 minutos de perfusão, sendo 24 homens e 16 mulheres. A idade dos pacientes variou de 45 a 79 anos, com a mediana de 67, havendo $12 \mathrm{com}$ idade superior a 70 anos. Coincidentemente em cada grupo, houve 6 operados com idade maior ou igual a 70 anos, assim como 12 homens e 8 mulheres. O peso variou de 45 a $94 \mathrm{~kg}$, com média de $70 \mathrm{~kg}$.

Todos foram submetidos à revascularização miocárdica pela primeira vez no Serviço de Cirurgia Cardíaca do Hospital Adventista Silvestre - Rio de Janeiro. Os pacientes foram divididos em grupos $A$ e B com 20 pacientes cada um.

- Grupo A: Perfusão com $\mathrm{pO}_{2}$ acima de $150 \mathrm{mmHg}$;

- Grupo B: Perfusão com $\mathrm{pO}_{2}$ abaixo de $100 \mathrm{mmHg}$. 
Moraes M C J, Moraes D J, Bastos E S, Murad H - Circulação extracorpórea com desvio veno-arterial e baixa pressão parcial de oxigênio. Rev Bras Cir Cardiovasc 2001; 16(3): 251-61.

\section{Grupo A}

Vinte pacientes operados, 12 homens e 8 muIheres, com idade variando entre 46 e 77 anos (média de 65 anos). O peso variou de 48 a $94 \mathrm{~kg}$ com média de $70 \mathrm{~kg}$.

A perfusão foi realizada com oxigenador de membrana DMG-3000, usando mistura de oxigênio com ar comprimido, sendo esta regulada em $40 \%$ de ar e $60 \%$ de oxigênio por meio de um misturador de gases ou "blender".

\section{Grupo B}

Constituído de 20 pacientes, 12 homens e 8 mulheres, com idade variando de 45 a 73 anos (média de 62 anos). O peso variou de 43 a $89 \mathrm{~kg}$ e média de $70 \mathrm{~kg}$.

Neste grupo, empregou-se o mesmo tipo de oxigenador, porém com oxigênio puro e desvio venoarterial regulado por meio de um torniquete, de modo que $40 \%$ do sangue venoso recirculava sem passar pelo oxigenador. Em 8 casos mediu-se o volume do desvio por meio de um fluxômetro eletromagnético "biopump" Medtronic.

Em todos os casos, foi monitorizada permanentemente a saturação de oxigênio da hemoglobina por meio de oxímetro "Bentley" acoplado em linha arterial e venosa.

Usou-se em todos pacientes bomba de rolete e oxigenador DMG. A perfusão foi normotérmica, e a hemodiluição com ringer e manitol ou ringer com haemacel e manitol. O fluxo de perfusão, nos 2 grupos de pacientes, foi obtido com drenagem de veias cavas e cânula única, tendo atingido em média 60 a $70 \mathrm{ml} / \mathrm{kg} / \mathrm{min}$ de peso corporal ou $2,2 \mathrm{l} / \mathrm{m} 2 / \mathrm{min}$ a $2,4 \mathrm{l} / \mathrm{m} 2 / \mathrm{min}$ de superfície corporal do paciente. A proteção miocárdica se deu com cardioplegia sangüínea 4:1 na raiz da aorta. Realizou-se o retorno arterial pela aorta em todos os pacientes (Figura 2).

A temperatura manteve-se entre $34^{\circ}$ a $36^{\circ} \mathrm{C}$, e o hematócrito variou entre 20 e $30 \%$, em todos os casos.

A anestesia foi realizada com fentanil, benzodiazepínico e curarização completa para se manter mínimo o consumo de oxigênio. No grupo com desvio veno-arterial, na fase final da perfusão, quando se acionava o permutador de calor para atingir-se a temperatura de $37^{\circ} \mathrm{C}$, reduziu-se o desvio em torno de 20 a 30\%. Além de determinação contínua da saturação arterial e venosa pelo oxímetro, foram realizadas gasometrias, com aparelho Ciba Corning 238, a cada 20 a 30 minutos, tanto do sangue venoso como do arterial, e monitoração contínua da pressão arterial.

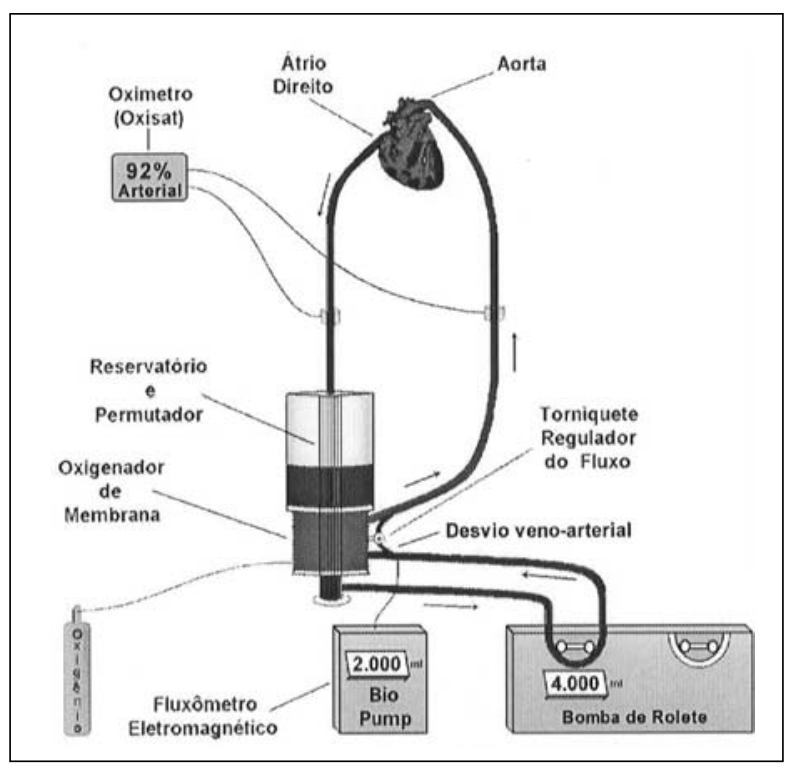

Fig. 2 - Esquema do circuito utilizado para os pacientes do Grupo B, do estudo clínico.

Em todas as operações foram solicitados como exames pré-operatórios: radiografia de tórax, eletrocardiograma, ecocardiograma e Doppler de carótidas (pacientes acima de 65 anos), além de exames de laboratório como: hemograma, glicose, uréia, creatinina, sódio, potássio, cálcio, magnésio, anti-HIV, antígeno Austrália, anti-hepatite C, coagulograma completo, tipagem sangüínea e gasometria arterial, obedecendo as técnicas habituais do laboratório de patologia clínica do Hospital Adventista Silvestre.

No período pós-operatório foram realizados radiografia de tórax, eletrocardiograma, ecocardiograma e exames de sangue (hemograma, coagulograma completo, bioquímica e gasometrias arterial e venosa). Foram observado9s o volume de drenagem de tórax a cada hora nas primeiras 48 horas do pós-operatório e a quantidade de bolsas de sangue total e ou hemoderivados transfundidos durante a hospitalização. Devido a essa monitorização, foi possível dar ao paciente maior segurança.

Durante a circulação extracorpórea, o oxigenador de membrana foi empregado nos 2 grupos.

Para a avaliação estatística, comparam-se os grupos de $\mathrm{pO}_{2}$ baixo e $\mathrm{pO}_{2}$ alto. Foi utilizado teste $\mathrm{t}$ de Student para verificar as diferenças entre as médias aritméticas; já nas variáveis em que os valores apresentavam grande variedade, foi empregado o teste não-paramétrico de Mann Whitney.

Adotou-se o nível de significância de 5\% de probabilidade. 
Moraes M C J, Moraes D J, Bastos E S, Murad H - Circulação extracorpórea com desvio veno-arterial e baixa pressão parcial de oxigênio. Rev Bras Cir Cardiovasc 2001; 16(3): 251-61.

TABELA 1

\begin{tabular}{lccccc}
\hline & \multicolumn{2}{c}{ VALORES DURANTE A PERFUSÃO, COMPARANDO O GRUPO I E O GRUPO II } \\
\cline { 2 - 3 } & \multicolumn{2}{c}{ MÉDIA GRUPO I } & & \multicolumn{2}{c}{ MÉDIA GRUPO II } \\
\cline { 2 - 3 } \cline { 5 - 6 } & ARTERIAL & VENOSO & & ARTERIAL & 7,31 \\
$\mathrm{pH}$ & 7,37 & 7,32 & 7,39 & 36,65 \\
$\mathrm{pCO}_{2}$ & 36,90 & 41,40 & 27,15 & 27,10 \\
$\mathrm{pO}_{2}$ & 109,05 & 38,20 & 58,35 & $-5,98$ \\
$\mathrm{BE}$ & $-3,75$ & $-3,64$ & $-5,56$ & $50 \%$ \\
$\mathrm{SAT}$ de $\mathrm{O}_{2}$ & $95 \%$ & $52 \%$ & & $89 \%$ & \\
\hline
\end{tabular}

TABELA 2

\section{MÉDIA REFERENCIAL DA GASOMETRIA ARTERIAL DOS VINTE CÃES DOS GRUPOS I E II ANTES DA PERFUSÃO, INCLUSIVE O HEMATÓCRITO}

\begin{tabular}{lcc}
\hline & \multicolumn{2}{c}{ MÉDIA S/ CEC } \\
\cline { 2 - 3 } & GRUPO I & GRUPO II \\
\cline { 2 - 3 } Hematócrito & $21,1 \%$ & $20,9 \%$ \\
$\mathrm{pH}$ & 7,46 & 7,43 \\
$\mathrm{pCO}_{2}$ & 30,10 & 24,40 \\
$\mathrm{pO}_{2}$ & 237,50 & 334,80 \\
$\mathrm{BE}$ & $-3,23$ & $-3,10$ \\
$\mathrm{SAT}$ de $\mathrm{O}_{2}$ & $99 \%$ & $99 \%$ \\
\hline
\end{tabular}

\section{RESULTADOS}

\section{Estudo Experimental}

No grupo I verificou-se que a função cardíaca manteve-se normal durante todo o período da perfusão. A pressão arterial permaneceu em torno de 60 a $80 \mathrm{mmHg}$. A dosagem dos gases revelou uma média de $\mathrm{pO}_{2}$ e saturação arterial e venosa em níveis aceitáveis, principalmente em relação à saturação venosa, que é a que melhor revela o balanço de oxigênio. A gasometria arterial foi colhida em 2 a 3 pontos da aorta torácica, sendo $0 \mathrm{pO}_{2}$ mais alto próximo à crossa. Deste modo, retirou-se amostra de sangue da aorta, sempre $3 \mathrm{~cm}$ acima do diafragma, onde a mistura do sangue estava mais homogênea.

No grupo II, uma vez fibrilado o ventrículo, a perfusão passou a ser feita com débito total, isto é, a soma das drenagens do átrio direito e do ventrículo esquerdo que, por sua vez, corresponde ao volume injetado pela segunda bomba na artéria pulmonar (Figura 1). Isto significa $50 \mathrm{ml} / \mathrm{kg} / \mathrm{min}$ ou metade do débito total que fica em torno de $100 \mathrm{ml} / \mathrm{kg} / \mathrm{min}$. A pressão arterial se manteve estável e, quando necessário, se acrescentou mais volume ao circuito da perfusão. A temperatura foi mantida pelo permutador de calor do reservatório de cardioplegia sangüínea DMG. No final de 30 minutos, faz-se cardioversão elétrica e, 5 minutos após, novamente induzia-se a fibrilação.
O resultado da média dos exames de gasometria,

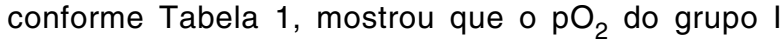
foi mais alto do que o do grupo II, tanto na gasometria arterial $\left(\mathrm{pO}_{2}=109 \times \mathrm{pO}_{2}=58\right)$ como na venosa $\left(\mathrm{pO}_{2}=38 \times \mathrm{pO}_{2}=36,6\right)$.

A saturação do grupo I foi mais alto quando comparado com grupo II também na gasometria arterial (SAT de $\mathrm{O}_{2}=95 \% \times$ SAT de $\mathrm{O}_{2}=89 \%$ ) e venosa (SAT de $\mathrm{O}_{2}=52 \% \times$ SAT de $\mathrm{O}_{2}=50 \%$ ).

Na Tabela 1 está demonstrada a média dos resultados de amostras de sangue arterial e venoso realizados durante a circulação extracorpórea dos grupos I e II.

A Tabela 2 demonstra os resultados médios do sangue arterial de exame de hematócrito e de gasometria, colhido 5 minutos antes de iniciar a circulação extracorpórea (CEC), dos grupos I e II.

\section{Estudo Clínico}

No grupo $\mathrm{A}$, de 20 pacientes com $\mathrm{pO}_{2}$ acima de $150 \mathrm{mmHg}$, houve perda sangüínea pelos drenos nas primeiras 48 horas, no CTI, de $16.590 \mathrm{ml}$ e com transfusões de 180 unidades de sangue ou hemoderivados.

Dentre os 20 pacientes do grupo $\mathrm{B}$, com $\mathrm{pO}_{2}$ abaixo de $100 \mathrm{mmHg}$, houve um total de $10.165 \mathrm{ml}$ de perda sangüínea pelos drenos nas primeiras 48 horas, com necessidade de transfusão de 63 unidades de sangue ou hemoderivados.

No grupo B, drenou-se apenas $65 \%$ do total drenado no grupo $A$. Também no grupo $B$ houve hemotransfusão de apenas $33 \%$ do total transfundido no grupo $A$.

\section{Não houve óbito hospitalar nos 2 grupos.}

Apesar de chamar o grupo $\mathrm{A}$ de $\mathrm{pO}_{2}$ acima de $150 \mathrm{mmHg}$, quando foi feito o estudo estatístico avaliando os valores de $\mathrm{pO}_{2}$ arterial, durante a circulação extracorpórea dos 20 pacientes deste grupo, encontrou-se um valor muito mais alto, ou seja, média 
Moraes M C J, Moraes D J, Bastos E S, Murad H - Circulação extracorpórea com desvio veno-arterial e baixa pressão parcial de oxigênio. Rev Bras Cir Cardiovasc 2001; 16(3): 251-61.

TABELA 3

\begin{tabular}{|c|c|c|c|c|c|}
\hline \multicolumn{6}{|c|}{$\mathrm{pO}_{2}$ ARTERIAL $(\mathrm{mmHg})$} \\
\hline VARIÁVEIS & GRUPOS & MÉDIA & DESVIO PADRÃO & TESTE & p \\
\hline \multirow[t]{2}{*}{ Total da amostra } & A & 192,44 & 48,92 & $t=-15,10$ & $\rho<0.01$ \\
\hline & B & 72,20 & 22,99 & & \\
\hline \multirow[t]{2}{*}{ Menor valor } & A & 163,55 & 46,32 & $t=-9,28$ & $\rho<0.01$ \\
\hline & B & 62,15 & 15,63 & & \\
\hline \multirow[t]{2}{*}{ Maior valor } & A & 222,25 & 37,34 & $t=-13,58$ & $\rho<0.01$ \\
\hline & B & 85,05 & 25,44 & & \\
\hline
\end{tabular}

de $\mathrm{pO}_{2}$ igual a $192,44 \mathrm{mmHg}$ (Tabela 3$)$. Em relação ao sangramento torácico constatou-se mediana de $760 \mathrm{ml}$ de drenagem sangüínea nas primeiras 48 horas de pós-operatório (Tabela 4) e obteve-se mediana de 9 unidades de transfusão de sangue ou hemoderivados (Tabela 5). Nessas mesmas tabelas foi surpreendentemente encontrado no grupo B apesar de classificá-lo como sendo $\mathrm{pO}_{2}$ abaixo de 100 $\mathrm{mmHg}$, uma média de $\mathrm{pO}_{2}$ que foi igual a 72,20 $\mathrm{mmHg}$. Já em relação ao sangramento torácico, obteve-se mediana de $495 \mathrm{ml}$ de drenagem sangüínea nas primeiras 48 horas e mediana de 3 unidades de transfusão de sangue ou hemoderivados.

Observamos que esses 40 pacientes toleraram bem a perfusão e tiveram evolução pós-operatória sem incidentes relacionados com perfusão. Os pacientes submetidos à circulação extracorpórea com baixo $\mathrm{pO}_{2}$ e desvio veno-arterial, tiveram menor sangramento e significativa redução no volume de transfusão de sangue e hemoderivados, no período hospitalar, quando comparados estatisticamente pelo teste não-paramétrico de Mann-Whitney com os do primeiro grupo (Tabelas 3, 4, 5, 6 e 7).

Apresenta-se a seguir os resultados avaliados estatisticamente.

Os valores medianos de dias internados em $\mathrm{pO}_{2}$ baixo e alto apresentam-se semelhantes. Em relação ao peso, idade e pressão arterial média durante a perfusão os valores médios comparados dos grupos $\mathrm{A}$ e $\mathrm{B}\left(\mathrm{pO}_{2}\right.$ alto e $\mathrm{pO}_{2}$ baixo) apresentaram diferenças não significativas.

Quanto ao tempo de perfusão, o valor médio no grupo $\mathrm{A}\left(\mathrm{pO}_{2}\right.$ alto $\left.=1: 34 \mathrm{~h}\right)$ apresenta valor mais elevado do que no grupo $\mathrm{B}\left(\mathrm{pO}_{2}\right.$ baixo $\left.=1: 19 \mathrm{~h}\right)$, sendo significativa a diferença.

A média do fluxo de perfusão é mais elevado no grupo $B\left(\mathrm{pO}_{2}\right.$ baixo $\left.=4,34 \mathrm{l} / \mathrm{min}\right)$ do que no grupo $\mathrm{A}\left(\mathrm{pO}_{2}\right.$ alto $\left.=3,74 \mathrm{l} / \mathrm{min}\right)$, sendo significativa a diferença.
TABELA 4

\begin{tabular}{lcc}
\hline \multicolumn{3}{c}{ SANGRAMENTO EM 48 HORAS $(\mathbf{m l})$} \\
\hline & GRUPO A & GRUPO B \\
\cline { 2 - 3 } Mediana & 760 & 495 \\
Valor mínimo & 350 & 190 \\
Valor máximo & 1660 & 950 \\
& $\mu=3,30$ & $\rho<0.01$ \\
\hline
\end{tabular}

TABELA 5

\begin{tabular}{lcc}
\hline \multicolumn{3}{c}{ TRANSFUSÃO DE HEMODERIVADOS (UNIDADE) } \\
\hline & Grupo A & Grupo B \\
\cline { 2 - 3 } Mediana & 9 & 3 \\
Valor mínimo & 4 & 1 \\
Valor máximo & 15 & 7 \\
& $\mu=4,71$ & $\rho<0.01$ \\
\hline
\end{tabular}

Tanto para menores como maiores valores em $\mathrm{pO}_{2}$ arterial, o grupo $\mathrm{A}\left(\mathrm{pO}_{2}\right.$ alto) apresenta médias mais elevadas do que grupo $\mathrm{B}\left(\mathrm{pO}_{2}\right.$ baixo), pois a média de $\mathrm{pO}_{2}$ arterial no grupo $A$, é mais elevado que a média do grupo $B$, e sendo significativa a diferença, conforme Gráfico 1 e Tabela 3, ou seja, a média do grupo $\mathrm{A}$ foi $\mathrm{pO}_{2}$ de $192,44 \mathrm{mmHg}$, enquanto que o grupo $B$ teve uma média de $72,20 \mathrm{mmHg}$.

Pode-se comparar no Gráfico $1 \mathrm{o} \mathrm{pO}_{2}$ durante a perfusão, com 3 variáveis de valores médios, mínimo e máximo, dos grupos $\mathrm{A}$ e $\mathrm{B}$ conforme Tabela 3. O grupo de pacientes com $\mathrm{pO}_{2}$ alto (grupo $A$ ), comparado com o grupo de $\mathrm{pO}_{2}$ baixo (grupo $\mathrm{B}$ ), apresentou os valores em média (Tabela 3 ).

O sangramento no grupo $\mathrm{A}\left(\mathrm{pO}_{2}\right.$ alto) em valor mediano é mais elevado do que no grupo $\mathrm{B}\left(\mathrm{pO}_{2}\right.$ baixo), sendo significativa a diferença, conforme se pode ver na Tabela 4 e no Gráfico 2.

O total de hemoderivados (concentrado de 
Moraes M C J, Moraes D J, Bastos E S, Murad H - Circulação extracorpórea com desvio veno-arterial e baixa pressão parcial de oxigênio. Rev Bras Cir Cardiovasc 2001; 16(3): 251-61.

TABELA 6

CARACTERÍSTICAS DOS GRUPOS A E B DO ESTUDO CLÍNICO

\begin{tabular}{lcccccc}
\hline & $\begin{array}{l}\text { IDADE } \\
\text { (ANOS) }\end{array}$ & $\begin{array}{c}\text { PESO } \\
\text { Kg }\end{array}$ & $\begin{array}{c}\text { PAM } \\
(\mathbf{m m H g})\end{array}$ & $\begin{array}{c}\text { FLUXO } \\
\text { (I/MIN) }\end{array}$ & $\begin{array}{c}\text { TEMPO } \\
\text { PERFUSÃO } \\
\text { (HORAS) }\end{array}$ & $\begin{array}{c}\text { DIAS } \\
\text { NO CTI }\end{array}$ \\
\hline Grupo A & 65,2 & 70,55 & 59,3 & 3,74 & $1: 34$ & 2,5 \\
Grupo B & 62,3 & 70,85 & 63,3 & 4,34 & $1: 19$ & 2,5 \\
$\rho$ & $<0.05$ & $<0.05$ & $<0.05$ & $<0.01$ & $<0.05$ \\
\hline
\end{tabular}

TABELA 7

\begin{tabular}{|c|c|c|c|c|c|}
\hline \multicolumn{6}{|c|}{ HEMODERIVADOS } \\
\hline VARIÁVEIS & GRUPOS & $\mathrm{X} / \mathrm{ME}$ & $\begin{array}{c}\text { S } \\
\text { (MÍN - MÁX) }\end{array}$ & TESTE & $\mathbf{p}$ \\
\hline \multirow[t]{2}{*}{ Sangue total } & $A$ & $X=1,46$ & 0,52 & $t=0,38$ & $\rho>0.05$ \\
\hline & B & $X=1,33$ & 0,58 & & \\
\hline \multirow[t]{2}{*}{ Concentrado de hemácias } & $A$ & $\mathrm{Me}=3,5$ & $(2-10)$ & $\mu=2,35^{\star \star}$ & $\rho<0.01$ \\
\hline & $\mathrm{B}$ & $\mathrm{Me}=2,0$ & $(1-4)$ & & \\
\hline \multirow[t]{2}{*}{ Plasma fresco } & A & $\mathrm{Me}=4,5$ & $(2-7)$ & $\mu=3,74^{\star *}$ & $\rho<0.01$ \\
\hline & B & $M e=2,0$ & $(1-4)$ & & \\
\hline \multirow[t]{2}{*}{ Total de unidades } & $A$ & $\mathrm{Me}=9,0$ & $(4-15)$ & $\mu=4,71^{* *}$ & $\rho<0.01$ \\
\hline & $\mathrm{B}$ & $\mathrm{Me}=3,0$ & $(1-7)$ & & \\
\hline
\end{tabular}

Média aritmética $(\mathrm{X})$, mediana (Me), desvio padrão (S), valores mínimos e máximos (quando há cálculo de mediana), testes (t de Student e não-paramétrico de Mann-Whitney), p (nível de significância).

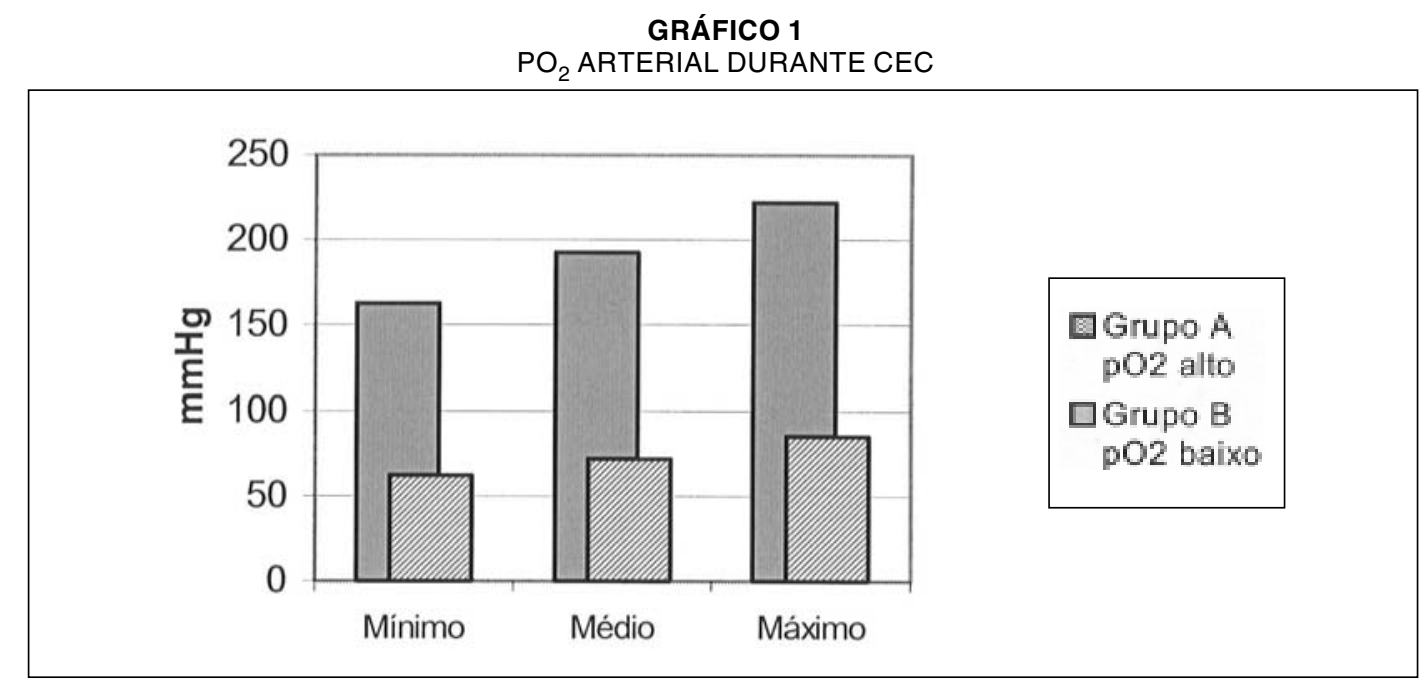

hemácias, concentrado de plaquetas e plasma fresco) apresenta mediana no grupo $\mathrm{A}\left(\mathrm{pO}_{2}\right.$ alto) mais elevada do que no grupo $\mathrm{B}\left(\mathrm{pO}_{2}\right.$ baixo), sendo significativa a diferença (Tabelas 5 e 7).
A mediana dos 20 pacientes do grupo $\mathrm{B}\left(\mathrm{pO}_{2}\right.$ baixo) transfundidos com hemoderivados foi de 3 unidades, enquanto a mediana dos outros 20 pacientes do grupo $\mathrm{A}\left(\mathrm{pO}_{2}\right.$ alto) foi de 9 unidades por paciente. 
GRÁFICO 2

MEDIANA DA QUANTIDADE DE SANGUE DRENADO NOS GRUPOS A E B, EM ml/48h
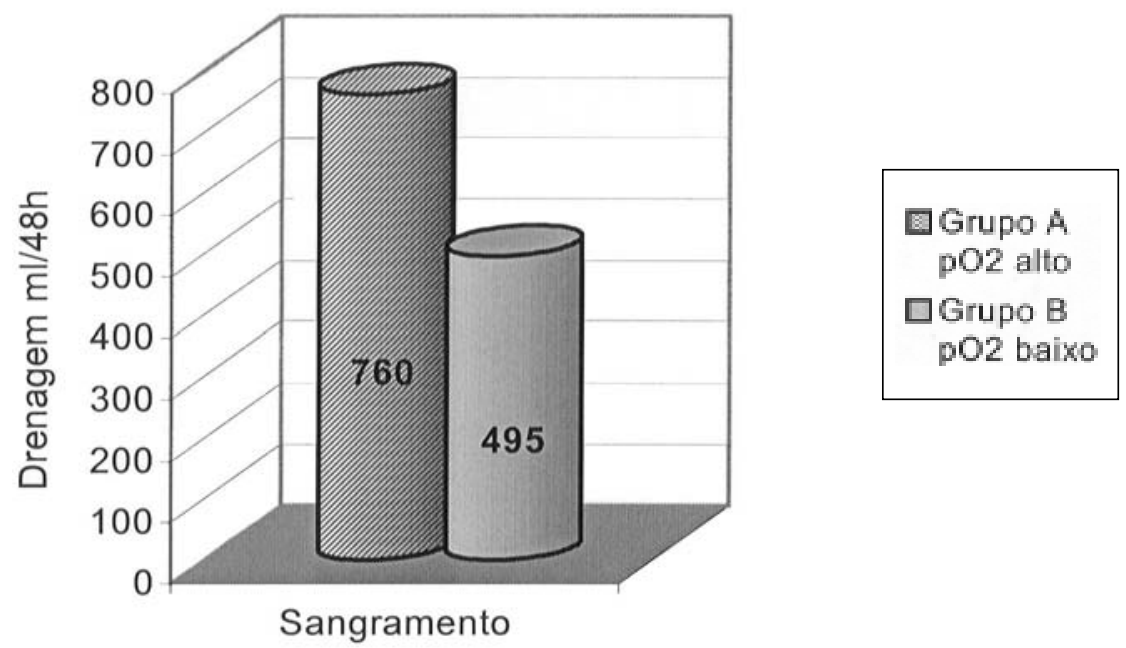

O Gráfico 2 demonstra a mediana dos grupos $A$ e $B$ quanto à hemotransfusão. Em 20 pacientes do grupo $\mathrm{B}$, com $\mathrm{pO}_{2}$ abaixo de $100 \mathrm{mmHg}$, houve drenagem com valor mediano de $495 \mathrm{ml} / 48 \mathrm{~h}$, enquanto que nos 20 pacientes do grupo $\mathrm{A}$, de $\mathrm{pO}_{2}$ alto, encontrou-se mediana de $760 \mathrm{ml} / 48 \mathrm{~h}$.

\section{COMENTÁRIOS}

Apesar do sucesso obtido com a grande maioria dos pacientes submetidos à operação cardíaca, boa parte da morbidade e talvez alguma porcentagem da mortalidade podem ser atribuídas a circunstâncias não-fisiológicas da circulação extracorpórea com exclusão cardiopulmonar(2).

Como se podem melhorar os efeitos danosos conseqüentes do emprego da circulação extracorpórea com a exclusão cardiopulmonar? Segundo BLACKSTONE et al.(3) e KIRKLIN et al. ${ }^{(1)}$ seriam esses os caminhos:

- Conseguir material que seja mais compatível do ponto de vista biológico com o sangue.

- Modificar o circuito usado para perfusão com exclusão cardiopulmonar.

- Desenvolver drogas que possam neutralizar os efeitos danosos conseqüentes da exposição do sangue ao circuito de circulação artificial.

Em relação ao item 1, vários materiais como policarbonato, polipropileno, acrílico, silicone, látex, polivinil, poliester, poliuretano e alumínio ajudam a construir melhores oxigenadores, sem, no entanto, formar o ambiente ideal para a passagem do sangue. O fato é que toda parte do circuito cria uma superfície hostil ao sangue. Este contato fora do ambiente do sistema vascular prejudica a sua integridade e estimula reações sangüíneas, incluindo a reação de coagulação. Atualmente, existe esforço para diminuir esse resultado com a aplicação de circuitos pré-heparinizados, que podem criar uma barreira biológica entre a superfície estranha do circuito e o sangue. Acrescentamos que a hemodiluição, (4-6) diminuindo a porcentagem de células sangüíneas, como hemácias, leucócitos e plaquetas que entram em contato com a superfície não-endotelial de material plástico, deve contribuir para diminuir os efeitos deletérios da circulação extracorpórea, sendo estas células os principais sinalizadores biológicos do sangue que reconhecem o meio artificial.

Trabalhos experimentais e clínicos controlados provaram haver redução em quase $100 \%$ das microembolias quando se substitui o oxigenador artificial pelo próprio pulmão $(7,8)$. Finalmente, os distúrbios de coagulação e conseqüente aumento do sangramento nos pacientes submetidos à circulação extracorpórea estão entre os problemas mais importantes e reconhecidos pelos cirurgiões desde o início do seu emprego ${ }^{(9)}$.

Propusemos e estudamos uma terceira hipótese: fluxo alto, porém com relativo baixo $\mathrm{pO}_{2}$ em normotermia. O objetivo foi diferenciar o que representa a perfusão tissular propriamente dita e o $\mathrm{pO}_{2}$ diminuído ou hipóxia. Em outras palavras, distinguir 
Moraes M C J, Moraes D J, Bastos E S, Murad H - Circulação extracorpórea com desvio veno-arterial e baixa pressão parcial de oxigênio. Rev Bras Cir Cardiovasc 2001; 16(3): 251-61.

hipoperfusão, vale dizer, isquemia de hipóxia. Já em 1982, GOLLAN ${ }^{(10)}$, pioneiro no estudo da fisiologia da circulação extracorpórea, enunciou os requisitos para uma perfusão ideal: fluxo alto, discreta hipercarbia, hemodiluição e discreta hipoxia $(12,12)$.

Os motivos alegados por GOLLAN (10) seriam que, dessa forma, se manteria melhor a perfusão dos órgãos internos, como cérebro, coração e rins devido à vasodilatação seletiva das artérias para as vísceras, que seriam assim melhor perfundidas do que a circulação periférica. Tal distribuição preferencial se observa, também, na circulação fetal. Por outro lado, admite-se que, dentre os fatores responsáveis pela reação inflamatória e morbidade, conseqüentes do uso mais ou menos prolongado de circulação extracorpórea, com exclusão cardiopulmonar, está o oxigenador (1).

É possível reduzir a saturação de hemoglobina do sangue, ao mesmo tempo em que se mantém alto o fluxo da perfusão tissular (13), e assim não haver tendência para metabolismo anaeróbico?

Naturalmente que dentre outros fatores a temperatura corporal seria dos mais importantes, pois com a redução da temperatura, teríamos uma proporcional redução do consumo de oxigênio (14). Os seguintes fatores diminuem o consumo de oxigênio, mesmo em normotermia:

- Sulfato de morfina 20 a $25 \%$

- Anestesia geral $25 \%$

- Respiração controlada e curarização $30 \%$

No estudo experimental nos limitamos a examinar animais (cães) com temperatura de $34^{\circ}$ a $36^{\circ} \mathrm{C}$, isto é, próximo do que se denomina perfusão normotérmica. Ao decidirmos usar o próprio pulmão do animal para oxigenar o sangue, em vez de um oxigenador artificial, tínhamos como objetivo observar apenas o limite mínimo de saturação do oxigênio da hemoglobina ou $\mathrm{pO}_{2}$ compatível com boa perfusão tecidual e saturação venosa acima de $50 \%$, sem a interferência dos fatores já referidos e que são relacionados ao uso do oxigenadores artificiais.

Todos os animais acordaram ao final da perfusão e não houve comprovação de tendência para discrasia sangüínea.

$\mathrm{Na}$ experimentação em cães, nossa proposta consistiu em criar um circuito de perfusão, que automaticamente só permitiria passar a metade do sangue venoso pelos pulmões. Nos animais em que foram mantidos os batimentos cardíacos e desviado todo o sangue de uma das cavas, deveria teoricamente haver um consumo pouco maior de oxigênio, pois o coração, mantendo a circulação pulmonar e parte da circulação sistêmica, deveria contribuir para aumento relativo do consumo de oxigênio. Isto pode- ria ser evidenciado pela menor saturação venosa da hemoglobina, quando comparado com o grupo em que a circulação tanto pulmonar como sistêmica era feita por meio de uma bomba mecânica (15).

Entretanto, pela comparação da média da saturação venosa verificada em cada grupo, não houve diferença significativa, sendo $52 \%$ em média no primeiro grupo e $50 \%$ no segundo. Em relação à saturação arterial, verifica-se que no grupo I ela esteve mais alta do que no grupo II, devido ao local de onde foi retirada a amostra de sangue na aorta (torácica). Isto é conseqüência de estar o coração batendo e ejetando o sangue que vem dos pulmões totalmente oxigenado. Assim, a saturação do grupo II representa melhor a real média quando se mistura a metade do sangue arterial com a metade do sangue venoso.

Como todos os animais foram submetidos à perfusão com hemodiluição e como o conteúdo de oxigênio do sangue depende do hematócrito, devemos nos lembrar de que paradoxalmente, pela diminuição da viscosidade sangüínea, mesmo com hematócrito mais baixo, pode haver até melhor transporte de oxigênio para os tecidos, segundo trabalho clássico de MESSMER (16). Nas temperaturas entre $32^{\circ} \mathrm{C}$ e $37^{\circ} \mathrm{C}$, a capacidade máxima de transportar oxigênio se dá com hematócrito de $30 \%$. Assim o hematócrito entre 20 e $30 \%$ pode até ser benéfico nestas circunstâncias. O melhor indicador para se avaliar o balanço do oxigênio é a saturação de oxigênio da hemoglobina venosa, que deve ficar acima de $50 \%$ ou $\mathrm{pO}_{2}$ acima de 27 ou $30 \mathrm{mmHg}$. O ideal é saturação venosa em torno de $70 \%$ ou $\mathrm{pO}_{2}$ de $35 \mathrm{mmHg}$.

Não encontramos na literatura consultada estudos feitos com esse mesmo circuito de perfusão, de modo que não foi possível comparar os nossos dados com os de outros.

$\mathrm{Na}$ observação clínica, em todos esses anos de circulação extracorpórea os oxigenadores e o sistema de monitorização melhoraram muito, porém o circuito básico pouco mudou. Assim, os 40 pacientes dos 2 grupos também foram perfundidos com a temperatura entre $34^{\circ}$ e $36^{\circ} \mathrm{C}$, e o que mais chamou atenção foi uma diferença estatisticamente significativa nas perdas sangüíneas pelos drenos de tórax nas primeiras 48 horas de pós-operatório, bem como quantidade de sangue e hemoderivados transfundidos em cada grupo de pacientes (17-19). O grupo A apresentou uma média de $\mathrm{pO}_{2}$ de $192 \mathrm{mmHg}$, e usou dois gases: ar comprimido e oxigênio. Entretanto, alguns hospitais não tem ar comprimido em rede ligado ao centro cirúrgico, mesmo os que o usam dependem de uma boa manutenção para evitar risco de contaminação. Outro problema não muito raro é que o misturador de gases ("blender") pode ficar desregulado. 
Moraes M C J, Moraes D J, Bastos E S, Murad H - Circulação extracorpórea com desvio veno-arterial e baixa pressão parcial de oxigênio. Rev Bras Cir Cardiovasc 2001; 16(3): 251-61.

No grupo B, mesmo usando oxigênio puro no oxigenador de membrana obteve-se média de $\mathrm{pO}_{2}$ igual a $72 \mathrm{mmHg}$ durante a perfusão; desviou-se o sangue venoso e sempre existiu a preocupação de diminuir o extenso contato do sangue com a superfície não endotelial dos oxigenadores e assim reduzir os efeitos deletérios ou inflamatórios da perfusão. Pois procurou-se passar até $50 \%$ do sangue venoso pelo desvio veno-arterial, usando sempre que possível fluxo alto. Devido a isso, o $\mathrm{pO}_{2}$ ficou mais baixo.

Em estudo comparativo, entre o oxigenador de membrana DMG modelo 3000 S (com desvio venoarterial) e o oxigenador de membrana Dideco (Itália) que foi realizado pela equipe do Professor Sérgio $A$. Oliveira (comunicação pessoal), em São Paulo, uma das conclusões a que chegaram foi que as hemácias dos pacientes perfundidos com desvio veno-arterial têm uma vida média mais longa do que as hemácias dos pacientes perfundidas pelo método clássico, isto é, sem desvio veno-arterial e com dois gases para alimentar o oxigenador. Talvez isso explique por que em nossa casuística os pacientes perfundidos sem desvio veno-arterial tiveram que receber maior quantidade de sangue e hemoderivados no período pós-operatório, ou seja, 9 unidades no grupo A contra 3 unidades no grupo $B$.

Das manifestações clínicas que caracterizam a agressão sofrida pelo organismo, quando submetido à circulação extracorpórea com oxigenação artificial, as alterações na cascata da coagulação foram reconhecidas desde o início da aplicação deste método à cirurgia cardíaca. Nos 20 pacientes submetidos à perfusão, com desvio veno-arterial, comparados aos outros 20 submetidos também à perfusão com dois gases no oxigenador, não houve diferença prática significativa quanto ao: tempo de perfusão, idade dos pacientes e temperatura durante a perfusão. Isto sugere que, o grupo $A, \operatorname{com} \mathrm{pO}_{2}$ mais alto, teve mais alterações na cascata da coagulação, pois está relacionado ao maior sangramento pelos drenos e, por conseqüência, mais transfusões de hemoderivados.

Face aos dados obtidos concluímos que esse método é no momento, sob este aspecto, mais adequado para a circulação extracorpórea.

RBCCV 44205-554

Moraes M C L, Moraes D J, Bastos E S, Murad H - Extracorporeal circulation with venous-arterial shunt and low oxygen partial pressure. Rev Bras Cir Cardiovasc 2001; 16(3): 251-61.

ABSTRACT: Purpose: This study is divided into 2 parts, an experimental study to establish a technique of extracorporeal circulation with low oxygen partial pressure and a clinical study to show the feasibility in humans.

Material and Methods: Experimental surgery with extracorporeal circulation was performed in 20 dogs divided into 2 groups of ten. In group I, cannulation was done first in the superior vena cava, then in the inferior vena cava, keeping normal heartbeat and breathing, controlled by a respirator and pure oxygen. After passing through a heat exchanger, the blood of each vena cava was injected in the femoral artery. Blood samples from the aorta were taken above the diaphragm in every 30 minutes to check gasometric values. In group II, the right atrium was drained and half of the blood injected in the pulmonary artery with another pump and picked up through the left ventricle to the reservoir that also works as a heat exchanger. The mixed blood (50\% arterial and $50 \%$ venous) was re-injected by another pump in the arterial circulation. The heart was maintained fibrillating and the breathing controlled by the respirator. In the clinical study, 40 patients were divided into 2 groups of 20 each. In group A the patients were bypassed in the conventional manner, that is, compressed air and oxygen in the oxigenator with high arterial $\mathrm{pO}_{2}$. In group $\mathrm{B}$, pure oxygen was used in the membrane oxigenator and venous-arterial shunt, performed between $40 \%$ to $50 \%$.

Results: In both groups, from a physiologic point of view there was shunting of $50 \%$ of venous blood to the arterial circulation and arterial blood flow was maintained high (around $100 \mathrm{ml} / \mathrm{kg} \backslash \mathrm{min}$ ). It was observed that the arterial $\mathrm{pO}_{2}$ in both groups remained between 50 and $100 \mathrm{mmHg}$ and venous saturation between 50 and $70 \%$. All animals woke up at end of the experiment. In the clinical study, the arterial $\mathrm{pO}_{2}$ was a low $60 \mathrm{mmHg}$. Comparison of the clinical results showed there was no mortality difference between both groups, but in the group $\mathrm{B}$, with the low $\mathrm{pO}_{2}$ and venous-arterial shunt, the post-operative bleeding was significantly smaller, having been used three times less blood and no need to use the blender.

DESCRIPTORS: Extracorporeal circulation, methods. Blood pressure, physiology. Oxygen, physiology. Venae cavae, surgery. Pulmonary artery, surgery. Oxygenators, membrane. 
Moraes M C J, Moraes D J, Bastos E S, Murad H - Circulação extracorpórea com desvio veno-arterial e baixa pressão parcial de oxigênio. Rev Bras Cir Cardiovasc 2001; 16(3): 251-61.

\section{REFERÊNCIAS BIBLIOGRÁFICAS}

1 Kirklin J K, George F J, Holman W - The inflamatory response to cardiopulmonary bypass. In: Gravlee G P, Davis R F, Utley J R, eds. Cardiopulmonary bypass: principles and practice. Baltimore: Williams and Wilkins, 1993: p.233-45.

2 Edmund Jr H L - Inflamatory response to cardiopulmonary bypass. Ann Thorac Surg 1998; 66(5suppl):12-6.

3 Blackstone $\mathrm{E} \mathrm{H}$, Kirklin $\mathrm{J} \mathrm{W}$, Stewart $\mathrm{R} \mathrm{W}$ et al. Damaging effects of cardiopulmonary bypass. In: Prostaglandins in clinical medicine. Chicago: Year Book Medical Publishers, 1982.

4 Moraes D J, Jazbik W, Coutinho H et al. - Hemodilution in extracorporeal circulation using plasma and dextran (macrodex) as diluents. J Cardiovasc Surg 1963; 4: 36-44.

5 Moraes D J, Jazbik W, Franco S - Perfusão prolongada com hemólise mínima: uso de plasma em substituição ao sangue do oxigenador. Rev Bras Cir 1960; 42: 120.

6 Moraes D J, Jazbik W, Franco S et al. - Estudo metabólico e hemodinâmico na circulação extracorpórea com exclusão cardiopulmonar. Hospital 1960; 57:201-23.

7 Marques E, Oshiro M S, Cestari I A, Hayashida S A, Leirner A A - Extracorporeal circulation with autogenous oxygenation: hemodynamic and gasometric parameters. Artif Organs 1995; 19: 470-4.

8 Moraes D J, Abilio F M, Cysne E et al. - Perfusion for myocardial mevascularization without an artificial oxygenator. Cardiovasc Bull Texas Heart Institute 1979; 6: 144.

9 Murad H - Complicações da circulação extracorpórea: introdução a circulação extracorpórea. Modelo Teórico № 1. Rio de Janeiro: Sociedade Brasileira de Circulação Extracorpórea, 1985.

10 Gollan F - Optimal blood perfusion. In: Zitwak R S \&
Jurado R A, eds. Care of the cardiac surgical patient New York: Appleton, 1982.

11 Hadjinikolaou L, Cohen A, Hafez H, Richmond W, Glenville B - Contribution of hyperoxia to lipid peroxidation in coronary artery operations: should we keep a low oxygen tension? J Thorac Cardiovasc Surg 1997; 113: 212-3.

12 Joachimsson P O, Sjöberg F, Forsman M, Johansson M, Ahn H C, Rutberg H - Adverse effects of hyperoxemia during cardiopulmonary bypass. J Thorac Cardiovasc Surg 1996; 112: 812-9.

13 Jazbik $A P$ - Nova técnica de perfusão retrógrada do miocárdio: estudo clínico e experimental. [Dissertação. Livre Docência]. Rio de Janeiro: Universidade Federal do Rio de Janeiro, 1970.

14 Cilley R E \& Bartlett R H - Extracorporeal life suport for respiratory failure. In: Gravlee G P, Davis R F, Utley $\mathrm{J} \mathrm{R}$, eds. Cardiopulmonary bypass principles and practice. Baltimore: Williams and Wilkins, 1993: p.660.

15 Mcgee W T, Veremakis C, Wilson G L - Clinical importance of tissue oxygenation and use of the mixed venous blood gas. Res Medica 1988; 4: 15.

16 Messmer K - Hemodilution. Surg Clin North Am 1975; 55: 659-78.

17 Borowiec J, Thelin S, Bagge L, Hultman J, Hansson H E - Decreased blood loss after cardiopulmonary bypass using heparin-coated circuit and $50 \%$ reduction of heparin dose. Scand $J$ Thorac Cardiovasc Surg 1992; 26: 177-85.

18 Kalter R D, Saul C M, Wetstein L, Soriano C, Reiss R F - Cardiopulmonary bypass: associated hemostatic abnormalities. J Thorac Cardiovasc Surg 1979; 77: 427-35.

19 Tanaka K, Takao M, Yada I, Yusa H, Kusagawa M, Deguchi $\mathrm{K}$ - Alterations in coagulation and fibrinolysis associated with cardiopulmonary bypass during open heart surgery. J Cardiothorac Anesth 1989; 3: 181-8. 